
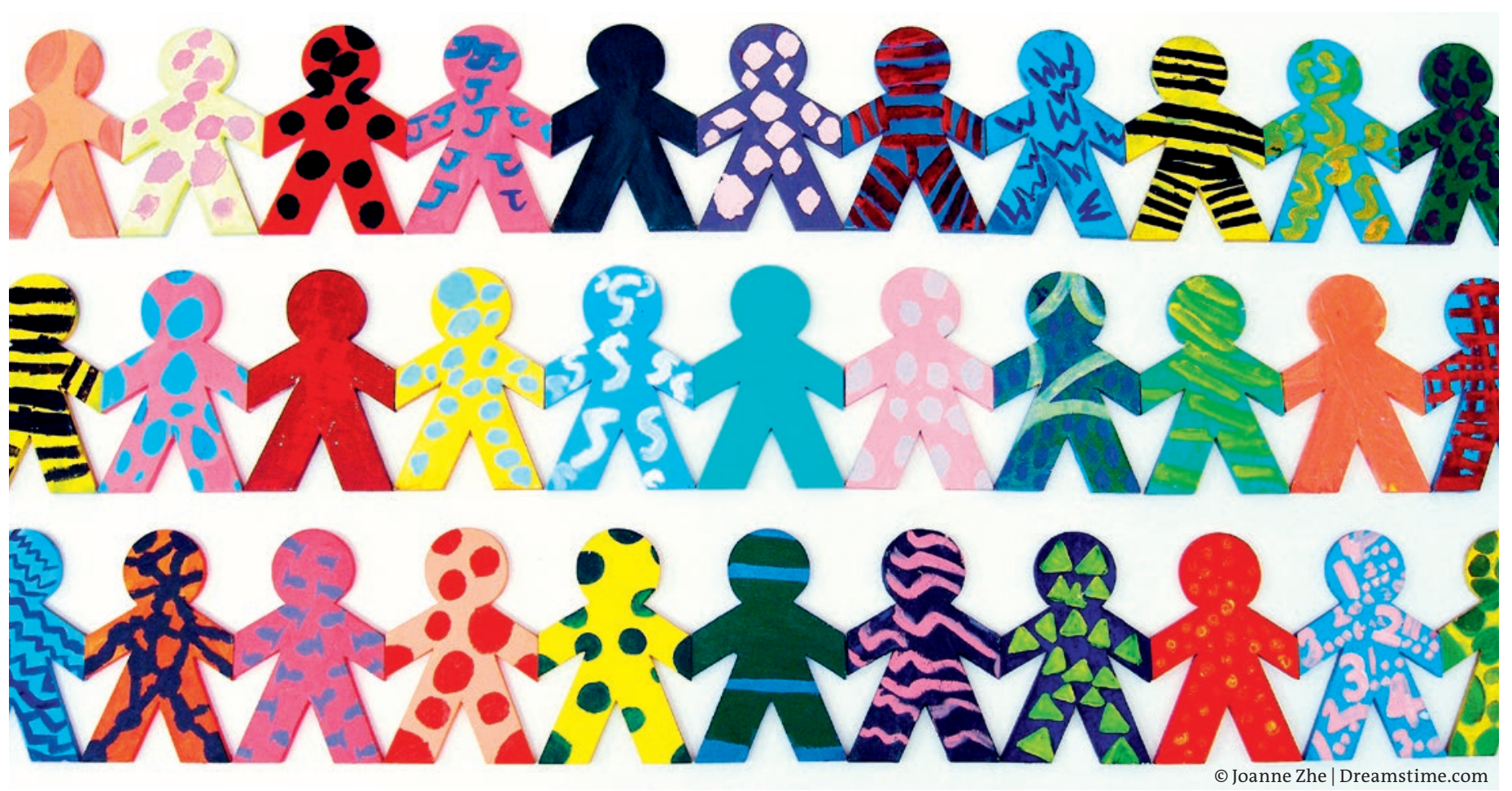

\title{
Approche interprofessionnelle pour les maladies rares
}

Jacqueline de Sá

Dre, Secrétaire générale, ProRaris

Le traitement des maladies rares, généralement complexes et chroniques, requiert une approche thérapeutique à plusieurs niveaux, qui doit tenir compte des aspects physiques, mais aussi psychologiques. La coordination entre tous les professionnels impliqués, avec la participation des patients, est cruciale.

Le terme «maladie rare» regroupe des affections très diverses. On en connaît jusqu'à 8000 dans le monde. Une maladie est dite rare si elle ne touche pas plus de cinq personnes sur 10000 . La plupart sont toutefois encore beaucoup moins fréquentes, chacune ne

\section{Série sur l'interprofessionnalité}

La collaboration entre les spécialistes de différentes professions de la santé est considérée comme un levier important pour relever les défis du système de santé. Où en est la Suisse dans ce domaine? Quels avantages apporte l'interprofessionnalité et quelles en sont les limites? Nous éclairons le sujet sous divers angles dans une série d'articles. concernant qu'un petit groupe de personnes, voire quelques individus isolés. En Suisse, selon les estimations de l'Office fédéral de la santé publique (OFSP), plus d'un demi-million de personnes, dont 350000 enfants et adolescents, sont atteintes d'une maladie rare.

\section{A la fois rares et complexes}

Quelque 80\% des maladies rares sont d'origine génétique. Ces anomalies génétiques peu fréquentes sont héréditaires ou résultent d'une mutation au cours du développement embryonnaire. Chez plus de la moitié des personnes concernées, les premiers symptômes 
apparaissent peu après la naissance ou dès la petite enfance. Les troubles peuvent être très variables. Beaucoup de ces maladies sont très complexes, touchent plusieurs organes et ont des répercussions sur l'ensemble de l'organisme. Elles sont chroniques, évolutives et entraînent souvent des handicaps graves et durables. La plupart sont en outre incurables. C'est un lourd fardeau, à la fois pour les malades et leur entourage familial. Seules quelques-unes se soignent efficacement. C'est par exemple le cas de certaines maladies métaboliques qui peuvent être traitées par thérapie enzymatique de substitution.

\section{Interdisciplinarité et interprofessionnalité}

Le traitement des maladies rares doit porter sur les aspects physiques, mais aussi psychologiques. En rai- son des troubles souvent multiples, la prise en charge de ces patientes et patients est un défi et nécessite un concept thérapeutique complexe, impliquant des expertes et experts de plusieurs disciplines et domaines de la santé. Comme de nombreuses maladies rares affectent différents organes à la fois, leur traitement

\section{Les maladies rares sont souvent complexes,} car elles touchent plusieurs organes et ont des répercussions sur l'ensemble de l'organisme.

exige une approche thérapeutique interdisciplinaire et des échanges réguliers entre les différents spécialistes médicaux et le médecin de famille. L'implication d'autres groupes professionnels est tout aussi essentielle pour la réussite du traitement. En font partie les physiothérapeutes, les orthophonistes et le personnel

\section{Interview de Surya Häne (29 ans), ingénieure en développement, Baden (AG)}

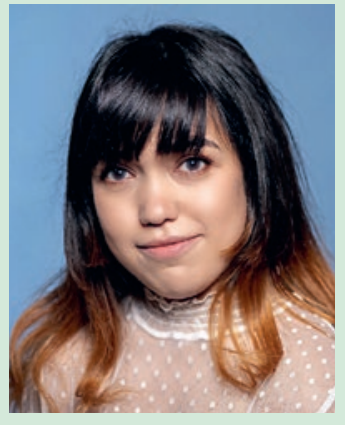

(C) mise à disposition par Surya Häne
Vous êtes atteinte de la maladie de MaroteauxLamy (MPS VI). Comment se manifeste-telle et quel traitement recevez-vous?

Il me manque une enzyme, ce qui entraîne des dépôts dans tout mon corps. Les symptômes sont apparus quand j'avais neuf ans. J'étais très souvent re-

froidie, j'avais des otites à répétition et une audition réduite. Je souffrais d'une apnée du sommeil sévère, avec jusqu'à 64 arrêts respiratoires par heure et de fortes crampes dans les mollets. Les symptômes se sont atténués avec la thérapie enzymatique de substitution que je suis depuis 2007. L'enzyme ne parvient toutefois que dans les régions bien vascularisées du corps. Au niveau des articulations et de la colonne vertébrale, les dépôts peuvent, au fil du temps, entraîner une compression des nerfs et une usure anormale, donc de fortes douleurs ou des troubles fonctionnels. Pour y remédier, on a recours à des opérations complexes et parfois risquées.

Quels sont les professionnels de la santé impliqués dans votre traitement?

La maladie affecte également l'étanchéité des valves cardiaques et la fonction pulmonaire. Un cardiologue et un pneumologue m'examinent régulièrement ces organes. Je suis également suivie par des spécialistes en métabolisme, chirurgie, orthopédie et neurologie. Je consulte aussi un physiothérapeute pour soulager mes douleurs dans les épaules et la nuque.

La collaboration entre les professionnels fonctionne-t-elle bien ou estimez-vous qu'elle pourrait être améliorée et, si oui, à quel niveau?

J'ai longtemps été soignée à l'Hôpital pédiatrique de Zurich, qui possédait l'expertise nécessaire et où les processus se déroulaient bien. Il y a deux ans, j’ai dû passer définitivement à la médecine de l'adulte. Je suis suivie par différents services de l'Hôpital universitaire de Zurich et de la Clinique universitaire de Balgrist. La prise en charge est moins optimale qu'avant. Si les échanges entre les établissements et les médecins fonctionnent - j'ai même eu droit à une consultation interdisciplinaire pour planifier la prochaine opération -, j'ai l'impression que c'est moi, en tant que patiente, qui dois me charger de la coordination. L'expertise est également moindre. Les échanges avec le physiothérapeute constituent la plus grosse pierre d'achoppement. Seule l'ordonnance lui est transmise, alors que des discussions entre professionnels seraient utiles. Je souhaiterais que les médecins aient plus de temps à consacrer aux patientes et patients. Ils devraient nous examiner en fonction de nos problèmes au lieu de taper des rapports sur leur ordinateur. C'est un peu caricatural, mais c'est ma perception. 
d'aide et de soins à domicile, des offices AI, des assurances-maladie, des organisations de patients spécifiques à la maladie, etc.

\section{Transition vers la médecine adulte}

Les enfants atteints de maladies rares bénéficient d'un suivi étroit. Grâce aux progrès de la médecine, ils atteignent de plus en plus souvent l'âge adulte. Cependant, pour de nombreuses maladies, la médecine adulte ne dispose pas encore du savoir-faire nécessaire, ce qui demande de mettre au point des traitements et des procédures. Pour les patients comme Surya Häne (voir interview p. 260), le passage à la médecine adulte et le changement de structure de soins sont souvent difficiles.

\section{Exemples d'interprofessionnalité vécue}

Les efforts actuels en vue d'aborder l'interprofessionnalité dès la formation initiale dans différents métiers de la santé et de l'encourager dans le travail quotidien méritent d'être salués. Voici deux exemples pratiques d'interprofessionnalité vécue de manière optimale dans le domaine des maladies rares:
1. Le centre de médecine génétique des Hôpitaux universitaires de Genève (HUG) recourt à des Genome boards. Ceux-ci réunissent des spécialistes de différentes disciplines, qui conjuguent leur expertise. Cette collaboration permet régulièrement de trouver des réponses à des cas non résolus.

2. Une patiente atteinte de deux maladies rares et de troubles liés à l'âge nous raconte comment fonctionne sa prise en charge interprofessionnelle. Son traitement est discuté avec tous les professionnels impliqués, ce qui garantit la continuité du flux d'informations. Ce sont les professionnels qui ont initié cette démarche. Les résultats d'examen et les propositions de traitement sont discutés avec la patiente. Toutes les personnes impliquées dans la prise en charge, comme le physiothérapeute, reçoivent un dossier complet. La patiente peut ainsi se concentrer sur son traitement et être certaine que tous les professionnels disposent des mêmes connaissances et s'engagent conjointement pour lui assurer un traitement optimal.

Nous aimerions que toutes les personnes atteintes de maladies rares bénéficient d'un tel environnement.

\section{Structures de soins pour personnes atteintes de maladies rares}

Les personnes atteintes de maladies rares doivent faire face à de nombreux défis, à commencer par l'établissement du diagnostic, qui prend souvent des années. A cela s'ajoutent des problèmes en termes de remboursement des traitements par les assurances sociales et la recherche d'offres de soutien appropriées, ou encore des difficultés lors de la formation et de l'exercice d'une activité professionnelle. La nécessité d'agir en faveur de la prise en charge des maladies rares a été reconnue, donnant le jour à un concept national dédié comportant 19 mesures en 2014.

Outre les professionnels de la santé, les patientes et patients ont été impliqués dans la planification de la mise en œuvre en faisant de ProRaris, l'association faîtière des personnes atteintes de maladies rares, un partenaire clé. ProRaris regroupe l'expertise des malades et l'intègre dans les divers projets de mise en œuvre. L'une des principales mesures est la désignation de centres de soins spécifiques afin d'améliorer la prise en charge des personnes atteintes de maladies rares.

La «Coordination nationale des maladies rares» (kosek) a été créée à cet effet en 2017. ProRaris en est membre fondateur et veille là aussi à ce que les patientes et patients soient impliqués dans le processus. La kosek distingue deux types d'offres de soins. Les Centres pour maladies rares sont des points de contact interdisciplinaires vers lesquels les patientes et patients dont le diagnostic n'est pas clairement établi peuvent se tourner pour effectuer des examens complémentaires et obtenir une réponse. Les centres travaillent de manière transversale et coordonnent l'expertise des spécialistes impliqués. Dès qu'un diagnostic est posé, les patientes et patients sont pris en charge par des centres de référence et des professionnels spécialistes de la maladie, organisés en réseaux nationaux. Ces centres appliquent les principes de la collaboration interprofessionnelle. 